\title{
From Uganda to Italy: a case of nephrotic syndrome secondary to Plasmodium infection, Quartan malarial nephropathy and kidney failure
}

\author{
Federico Gentile², Marida Martinoํㅡㄴ Luisa Santangelo ${ }^{1}$, Paolo Giordano ${ }^{4}$, \\ Diletta Domenica Torres ${ }^{1}$, Vincenza Carbone ${ }^{1}$, Anna Maria Di Palma ${ }^{3}$, \\ Michele Rossini $^{3}$, Loreto Gesualdo ${ }^{3}$, Paola Giordano ${ }^{5}$, Mario Giordano ${ }^{1}$ \\ ${ }^{2}$ Post Graduated School in Pediatrics, University of Bari Aldo Moro, Bari; ${ }^{1}$ Pediatric Nephrology and Dialysis Unit, Pediatric \\ Hospital Giovanni XXIII, Bari; ${ }^{4}$ Post Graduated School in Pediatrics, University of Genoa; ${ }^{3}$ Department of Emergency and \\ Organ Transplantation - Nephrology, Dialysis and Transplantation Unit, University of Bari Aldo Moro, Bari; ${ }^{5}$ Department \\ of Biomedical Science and Human Oncology-Pediatric Unit, Bari, Italy. E-mail: mario.giordano@policlinico.ba.it \\ Received: 13th March 2018, Revised: 7th August 2018, Accepted: 27th October 2018
}

SUMMARY: Gentile F, Martino M, Santangelo L, Giordano P, Torres DD, Carbone V, Di Palma AM, Rossini M, Gesualdo L, Giordano P, Giordano M. From Uganda to Italy: a case of nephrotic syndrome secondary to Plasmodium infection, Quartan malarial nephropathy and kidney failure. Turk J Pediatr 2019; 61: 776-779.

Malaria (M), the first parasitic infection, is sometimes associated with nephrotic syndrome (NS) in tropical areas. Kidney involvement during quartan malaria is represented by immune-complex mediated glomerulonephritis (GN). Generally, NS develops several weeks after onset of quartan fever and its clinical course proceeds slowly to end-stage kidney disease (ESKD) even after eradication of the infection. We describe a case of Plasmodium malariaeassociated nephrotic syndrome and chronic proliferative glomerulopathy in a boy from Uganda. Renal biopsy revealed chronic proliferative GN with capillary wall thickening producing a double contour, segmental sclerosis and tubular atrophy. Blood Giemsa smear contained rare ring-form trophozoites and gametocytes of Plasmodium spp. This case highlights the importance of obtaining remote travel histories from immigrants presenting with nephrotic syndrome especially due to the current immigration crisis in Europe. Malaria has low prevalence or less known in our continent and requires more medical attention by European doctors.

Keywords: proliferative glomerulopathy, malaria, nephrotic syndrome, renal failure, quartan malarial nephropathy.

Malaria (M), the first parasitic infection, is sometimes associated with nephrotic syndrome (NS) in tropical areas. Plasmodium malariae is unique among the plasmodia in which subclinical parasitemia may persist for decades. Chronic P. malariae infection was linked to nephrotic syndrome in children in the 1960s and subsequently attributed to immune complex basement membrane nephropathy. Generally, NS develops several weeks after onset of quartan fever and its clinical course proceeds slowly to end-stage kidney disease (ESKD) even after eradication of the infection. ${ }^{1}$ We describe a case of $P$. malariae-associated nephrotic syndrome and chronic proliferative glomerulopathy in a boy from Uganda.

\section{Case Report}

A 17-year-old Ugandan boy was transferred

This was presented at the 50th European Society of Pediatric Nephrology Meeting, 6-9 September 2017, Glasgow, Scotland, United Kingdom. 
in Italy, as part of a humanitarian project, to treat ESKD onset 13 months before as NS. In Uganda, the patient received steroids and immunosuppressive therapy with poor results and some occasional dialysis sessions due to socioeconomic reasons. His past medical history was significant only for malaria but overall was partially unknown. On admission to our department, the boy weighed $49,5 \mathrm{~kg}$ and was $154 \mathrm{~cm}$ in height. Clinical examination showed a pale, febrile and edematous boy with hypophonesis in the right hemithorax and a malodorous purulent secretion from exit site of central venous catheter (CVC). Laboratory tests revealed massive proteinuria $(11,9 \mathrm{~g} / 24 \mathrm{~h}$ or $341,9 \mathrm{mg} / \mathrm{m}^{2} / \mathrm{h}$ ) and laboratory findings of kidney failure: serum creatinine $4,0 \mathrm{mg} / \mathrm{dl}$, glomerular filtration rate $21 \mathrm{ml} / \mathrm{min} . / 1.73 \mathrm{~m}^{2}$, BUN $69 \mathrm{mg} / \mathrm{dl}$, albumin 10,2 g/l and uric acid $4,8 \mathrm{mg} / \mathrm{dl}$. The arterial blood gas showed $\mathrm{pH}$ 7.25 with $\mathrm{HCO} 318.7 \mathrm{mmol} / \mathrm{l}$, hypocalcemia $\left(\mathrm{Ca}^{2+} 7,2 \mathrm{mg} / \mathrm{dl}\right)$, hypokalemia $\left(\mathrm{K}^{+} 3,4 \mathrm{mEq} / \mathrm{l}\right)$ and normal values for $\mathrm{Na}^{+} 139 \mathrm{mEq} / \mathrm{l}, \mathrm{Cl}^{-} 106$ $\mathrm{mEq} / \mathrm{l}, \mathrm{P} 4,2 \mathrm{mg} / \mathrm{dl}$. There was also leukocytosis
(WBC 26,1 x 103/ $\mu \mathrm{l}$ ), anemia (Hb 8,44 g/dl), and high serum C-reactive protein $(213 \mathrm{mg} /$ dl), C3 0,95 g/l, C4 0,41 g/l. He also had a condition of secondary hyperparathyroidism (PTH $390 \mathrm{pg} / \mathrm{ml}$ ) with the need for vitamin D supplementation.

Culture's catheter exit-site exudate was positive for Staphylococcus aureus. Renal ultrasound showed small and hyperechoic kidneys but no stones or masses; chest CT scan showed copious left pleural effusion with partial left lung collapse. Renal biopsy revealed chronic proliferative GN with capillary wall thickening producing a double contour, segmental sclerosis and tubular atrophy. (Fig. 1) Immunofluorescence staining identified granular deposits of IgA and C3 on mesangium. Evaluation for secondary causes such as the viral hepatitis, human immunodeficiency virus (HIV), syphilis, tuberculosis, malignancy, auto-immune diseases, toxic exposure and medication was not fruitful. Given the patient's history of malaria, we did microscopic examination of

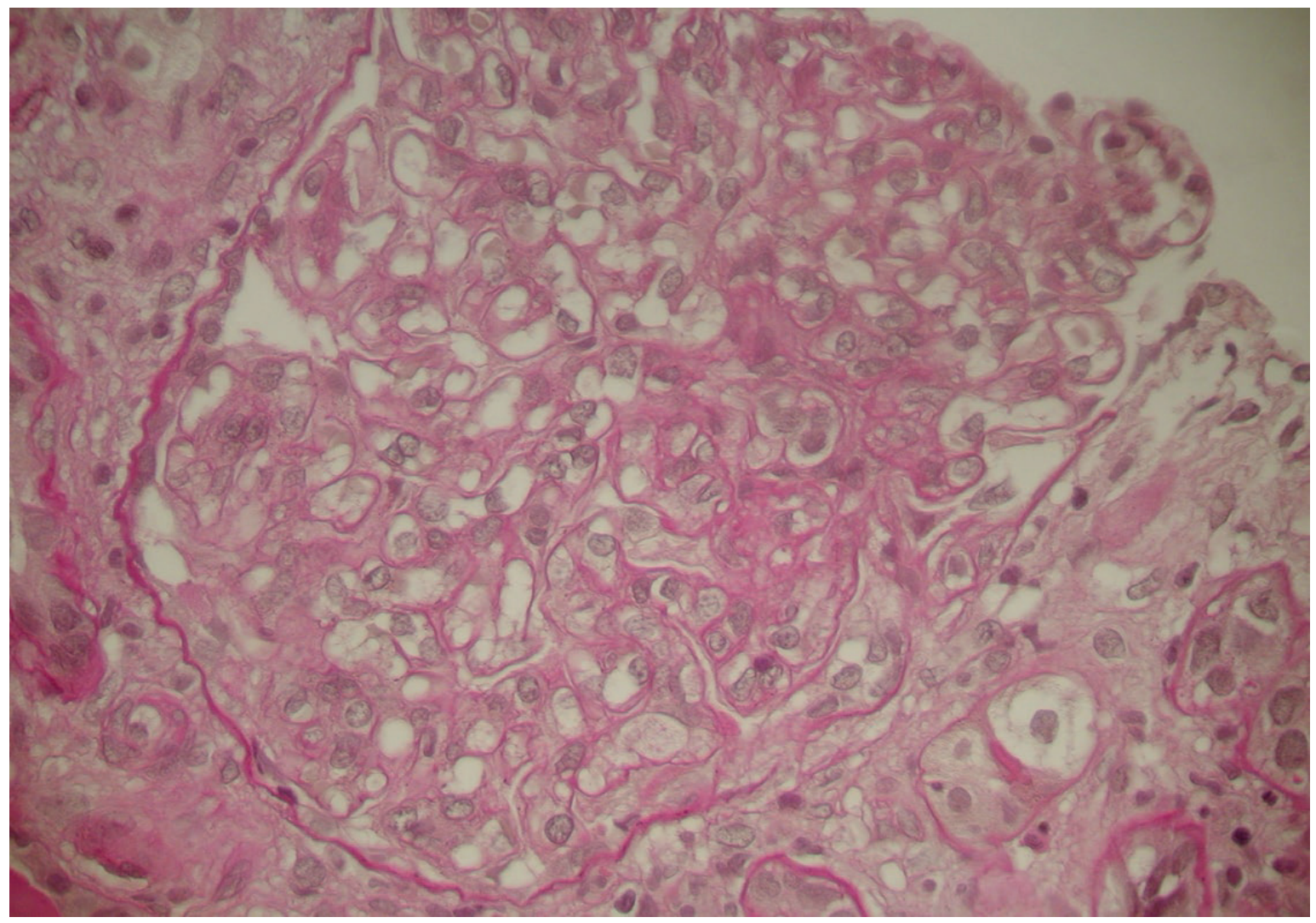

Fig. 1. A glomerulus showing endocapillary proliferation with segmental double contours of the glomerular basement membrane with cellular interposition. (PAS, x400). 
the patient's Giemsa-stained blood smears which showed rare ring-form trophozoites and gametocytes of Plasmodium spp. The patient was treated with atovaquone/ proguanil for 3 days and with meropenem + vancomycin i.v. for one week. The pleural effusion required the placement of pleural drainage for 3 weeks. histological findings evidenced sclerosis in a high number of glomeruli, for this reason immunosuppressive therapy was avoided. Thanks to intensive therapy (antimalaric and antibiotics drug, pleural drainage) and to the improved conditions of assistance, his general condition improved while kidney function improved finally and the boy was dismissed after 45 days of hospitalization and was enrolled into a dialysis/transplantation program requiring dialysis three times in a week. Written informed consent was obtained from the patient and his family for publication of this case report.

\section{Discussion}

The first definite causal relationship between P. malariae and NS was reported by Giglioli in 1930. P. malariae and P. falciparum are commonly associated with glomerular disease although a few cases with abnormal renal function have been associated with $P$. vivax infection. Chronic and progressive glomerulopathy in $P$. malariae (quartan malaria) and malaria acute renal failure (MARF) are considered as the two major renal disorders associated with malaria. ${ }^{1}$ A statistically significant relationship between malaria infection and the NS was shown by Gilles and Hendrickse ${ }^{4}$ in nephrotic children. Of these, $88 \%$ tested positively for malaria compared to $24 \%$ in non-nephrotic children. The corresponding rates for $\mathrm{P}$. falciparum were $62 \%$ in nephrotic children and $70 \%$ in nonnephrotic children. Kibukamusoke ${ }^{5}$ showed that quartan malaria NS (QMNS) was found more frequently during the rainy season than during the dry season, affecting mostly children at a peak age of 5 and 8 years. The main clinical symptom of QMNS was generalized edema. Analysis of proteinuria revealed nonselective glomerular proteinuria in most cases. The prognosis of QMNS was poor: usually it is steroid resistant and unresponsive to azathioprine and cyclophosphamide.
The 5-year patient survival rate was $60 \%$. On light microscopy, the basic glomerular lesion consisted of capilliary wall thickening involving the subendothelial aspect of the basement membrane, producing either a double contour ora plexiform arrangement of PAS-positive argyrophilic fibrils. On immunofluorescence, granular deposits of IgG, IgM and C3 complement were described in the glomeruli and deposition of malarial antigens in the glomeruli of nephrotic humans was generally not shown in renal biopsies. Renal biopsy in Ugandan patients with QMNS revealed proliferative glomerulonephritis in the majority of patients. In Nigeria, Edington and Mainwaring found mostly focal and segmental glomerulosclerosis. ${ }^{2}$ In summary, the role of Plasmodium in the aetiology of human glomerulonephritis is based on circumstantial clinical and epidemiological evidence and on renal biopsies showing granular immune deposits in the glomeruli. In the light of confirmed P. malariae infection by blood smear and histological findings with absence of other alternative causes of proliferative GN, we believe this case could be consistent with QMN. Actually, the association between Plasmodium infection and NS remains controversial. It was never explained why the quartan malaria NS was associated with proliferative glomerular lesions in Ugandan patients, whereas Nigerian patients had a more membranous type of lesion. ${ }^{4}$ Ethiopatogenetic difference is not explained by the immune complex theory. ${ }^{3}$ Malaria infection has been reported with increasing frequency associated with severe morbidity in immunocompromised renal transplant recipients who receive their allografts from infected donors living in high-risk areas. The true incidence and severity of malaria among the post-transplant recipients is not completely defined, routine malaria prophylaxis among renal transplant recipients should be provided especially in malaria endemic zones. Early detection and appropriate treatment improves the prognosis of this potentially life-threatening complication in the post-transplant period. Immunosuppression appears to increase the risk of malaria complications particularly in renal transplant recipients. Cyclosporine (Cs) is usually used as immunosuppressant in renal transplant recipients to improve 
graft survival and prevent acute and chronic rejection after kidney transplantation. Fortunately, besides the immunosuppressive effect of CsA, the drug has inhibitory activity against intraerythrocytic growth of the malaria parasite. Thus, immunosuppressive therapies can act synergistically with antimalarial agents to fight malaria in renal transplant recipients.

However, this case highlights the importance of obtaining remote travel histories from immigrants presenting with NS especially due to the current immigration crisis in Europe. Malaria has low prevalence or is less known in our continent and requires more medical attention by European clinicians. ${ }^{6}$ Furthermore, kidney involvement during malaria is considered as a negative prognostic factor. $^{7}$ Asymptomatic immigrants from more common endemic malaria areas (West Africa, Southeast Asia, Eastern Indonesia) or other people with anamnestic history or malaria need to undergo clinical surveillance in order to avoid late recrudescence or complications like GN, with more sensitive diagnostic tools.

\section{REFERENCES}

1. Elsheikha HM, Sheashaa HA. Epidemiology, pathophysiology, management and outcome of renal dysfunction associated with plasmodia infection. Parasitol Res 2007; 101: 1183-1190.

2. Edington GM, Mainwaring AR. Nephropathies in West Africa. In: Mostofi FK, Smith DE (eds). The Kidney. Baltimore: Williams and Wilkins, 1966: 488.

3. Ehrich JH, Eke FU. Malaria-induced renal damage: facts and myths. Pediatr Nephrol 2007; 22: 626-637.

4. Gilles HM, Hendrickse RG. Nephrosis in Nigerian children: role of Plasmodium malariae, and effect of antimalarial treatment. Br Med J 1963; 2: 27-31

5. Kibukamusoke JW, Hutt MSR, Wilks NE. The nephrotic syndrome in Uganda and its association with quartan malaria. QJM Int J Med 1967; 36: 393408

6. Eiset $\mathrm{AH}$, Wejse C. Review of infectious diseases in refugees and asylum seekers-current status and going forward. Public Health Rev 2017; 38: 22.

7. Abstracts from the 2017 Society of General Internal Medicine Annual Meeting. J Gen Intern Med 2017; 32(Suppl 2): 83-808. (Slinchenkova O, Coyle C. Severe malaria: how to predict mortality-Scientific Abstracts S589-S590). 